\title{
La représentation de la diversité culturelle québécoise dans la publicité télévisée francophone
}

A representação da diversidade cultural do Québec na publicidade francófona televisiva

FARRAH BÉROUBÉ béroubé.farrah@courrier.uqam.ca

\begin{abstract}
Résumé
Dans les années 50 , les chercheurs ont observé dans les médias de masse une représentation inadéquate de la diversité culturelle aux États-Unis et au Canada. Par exemple, des groupes étaient sousreprésentés dans les médias par rapport à leur nombre dans la population et des représentations perpétuaient des stéréotypes raciaux. Depuis, le «visage» des pays occidentaux ne cesse de se diversifier culturellement. Selon le recensement de 2001, le Canada compte 5,4 millions d'immigrants, soit 18,4 \% de sa population totale, une réalité palpable dans les grandes villes comme Montréal. Qu'en est-il aujourd'hui de la représentation de la diversité culturelle québécoise dans les médias francophones? Cet article, qui a pour perspective communicationnelle les médias et la diversité culturelle, présentera les résultats d'une analyse de contenu réalisée en 2003 sur la représentation de cette diversité québécoise dans la publicité télévisée francophone. Les résultats montrent, entre autres, que les représentations véhiculent des stéréotypes raciaux, qu'il y a sur-représentation de certains groupes, alors que d'autres groupes sont sous-représentés.
\end{abstract}

\section{Introduction}

C'est aux États-Unis dans les années 1950 que des premiers chercheurs étudient et dénoncent la façon dont sont représentés les Noirs dans les médias (Shuey, King, et Griffith 1953; Colfax et Sternberg 1972; Culley et Bennett 1976). Ils affirment que ces représentations, lorsqu'elles existent, sont racistes. Cette idée est renforcée et approfondie avec l'avènement des Cultural Studies lorsque sont étudiées les problématiques identitaires aux États-Unis, au Canada et en Grande-Bretagne. À la fin des années 1990, les chercheurs ont observé une amélioration puisque les prises de position ouvertement racistes dans les médias ont diminué, la diversité culturelle y est de plus en plus représentée et les stéréotypes raciaux y sont de moins en moins perpétués (Cottle 2000; Fleras et Lock Kunz 2001; Fleras 2006). Cependant, ces chercheurs ne crient pas victoire puisqu'ils imputent cette évolution à la montée de ce qu'ils appellent le nouveau racisme ou le racisme démocratique, soit cette idéologie qui préconise la promotion d'idéaux démocratiques comme l'égalité et l'équité, mais où semblent coexister des attitudes et des comportements contradictoires, au nombre desquels se trouve la discrimination des minorités ethniques (Mahtani 2001). Dans la littérature scientifique récente, on souligne donc toujours que la diversité culturelle n'est pas traitée à sa juste valeur dans les médias occidentaux.

\section{Problématique et questions de recherche}

Qu'en est-il au Québec? Comment sont représentés les Québécois d'origines ethniques différentes dans les médias francophones? En 1993, le cher- 
cheur Pierre-André Tremblay relevait une pauvreté d'études sur la question de la représentation de la diversité culturelle québécoise dans les médias francophones, pauvreté qui persiste encore.

Afin de contrer cette lacune, nous avons mené en 2003 une recherche de deuxième cycle où nous posions la question suivante: «les représentations de la diversité culturelle québécoise diffusées dans les médias francophones reflètent-elles les réalités quantitative et qualitative de la présence ethnique au Québec?» Nous avons choisi de procéder par analyse de contenu, méthode très utilisée en recherche sur les médias et les représentations de la diversité culturelle, afin de pouvoir éventuellement comparer nos résultats avec les recherches antérieures et étrangères. Aussi, nous avons décidé de limiter notre objet d'analyse à la publicité télévisée pour la projection culturelle qu'offre ce média. Ainsi, le corpus de notre étude était composé de toutes les publicités diffusées sur une semaine sur les quatre grandes chaînes de télévision québécoise francophone, soit Télé-Québec, Radio-Canada, TVA et TQS. Plus spécifiquement, nous avons cherché à savoir si les représentations des Québécois d'origines ethniques différentes étaient proportionnelles à leur nombre dans la société et si elles perpétuaient des stéréotypes raciaux. Afin de répondre à ces questions, nous avons, dans un premier temps, repérés toutes les publicités représentant au moins un Québécois d'origine ethnique différente, et ce, de manière visuelle, imagée ou auditive. La diversité culturelle ainsi représentée a été compilée sous six grandes familles ethniques correspondant plus ou moins aux grandes régions ou continents du globe, soit les Latino-américains, les Moyen-orientaux, les Asiatiques, les Nord-Américains (autres que les Québécois dit «de souche»), les Euro- péens et les Africains. Les identités et les appartenances étant multiples, cette classification n'avait pour but que de tracer quelques grandes lignes entre les différentes et combien nombreuses origines ethniques pour le bien de l'exercice. Ce repérage nous a permis une toute première analyse, soit celle de la représentation quantitative de la diversité culturelle québécoise dans les publicités télévisées francophones. Dans un second temps, nous avons revisionné les mêmes publicités en analysant qualitativement les représentations.

Cet article présente les principaux résultats de cette étude soit, dans un premier temps, qu'il y avait, dans les publicités analysées, une sur-représentation de certains groupes (les Noirs et les Asiatiques), alors que d'autres groupes étaient sous-représentés (les Amérindiens, les Canadiens anglophones, etc.). Enfin, l'analyse qualitative nous a permis l'identification de représentations entretenant des stéréotypes raciaux.

\section{La représentation quantitative de la diversité culturelle québécoise dans les publicités télévisées francophones}

Le tableau 1 présente le nombre de publicités diffusées sur une semaine sur les quatre grandes chaînes de télévision francophone québécoise et qui ont été analysées. Au total, 5699 publicités ont été diffusées et de ce nombre 1007 publicités représentaient au moins un Québécois d'origine ethnique différente, soit $17,7 \%$ de l'ensemble des publicités.

Le tableau 2 présente les grandes familles ethniques qui ont été représentées dans les publicités analysées. Ainsi, 864 publicités ont représenté au moins un Québécois appartenant à la grande famille africaine ce qui corres- 
Tableau 1: Publicités représentant des ethnies par chaîne de télévision québécoise francophone.

\begin{tabular}{l|cccc|c}
\hline \multirow{2}{*}{\multicolumn{1}{c|}{ Genre de publicité }} & \multicolumn{4}{c|}{ Chaînes de télévision } & \multirow{2}{*}{ Total } \\
\cline { 2 - 5 } & SRC & TVA & TQ & TQS & \\
\hline Publicités sans ethnie & 1060 & 1424 & 689 & 1519 & 4692 \\
Publicités avec ethnies & 252 & 340 & 159 & 256 & 1007 \\
Total & 1312 & 1764 & 848 & 1775 & 5699 \\
\hline $\begin{array}{l}\text { Proportion (\%) de publicités avec ethnies sur le total des publicités diffu- } \\
\text { sées sur chacune des chaînes }\end{array}$ & 19,2 & 19,3 & 18,8 & 14,4 & 17,7 \\
\hline
\end{tabular}

Tableau 2: Nombre de publicités représentant les grandes familles ethniques.

\begin{tabular}{|c|c|c|}
\hline Familles ethniques & Nombre de publicités & $(\%)$ \\
\hline Latino-américains & 36 & 0,6 \\
\hline Moyen-orientaux & 103 & 1,8 \\
\hline Asiatiques & 339 & 5,9 \\
\hline Nord-américains (autre que Québécois) & 21 & 0,4 \\
\hline Européens & 126 & 2,2 \\
\hline Africains & 864 & 15,2 \\
\hline Total & 1007 & 17,7 \\
\hline
\end{tabular}

Tableau 3: Pourcentages des grandes familles ethniques dans la population du Québec et des publicités représentant ces familles

\begin{tabular}{lcc}
\hline \multicolumn{1}{c}{ Familles ethniques } & Nombre au Québec (\%) & Nombre de publicités (\%) \\
\hline Latino-américains & 0,1 & 0,6 \\
Moyen-orientaux & 0,3 & 1,8 \\
Asiatiques & 1,7 & 5,9 \\
Nord-américains & 1,8 (Amérindiens) & 0,4 \\
(autres que Québécois) & 3,1 (Anglophones) & 2,2 \\
Européens & 0,6 & 15,2 \\
Africains & 2,1 & 17,7 \\
\hline Total & 9,4 & \\
\hline * CANADA, STATISTIQUE CANADA, Bienvenue à Statistique Canada, [En ligne], 15 juillet 2003, http://www.statcan.ca/(Page consultée le 15 juillet 2003).
\end{tabular}

pondait à 15,2\% des publicités analysées. Selon le tableau 2, les grandes familles ethniques qui ont été le plus représentées dans les publicités analysées étaient les Québécois d'origines asiatique et africaine. Qu'en est-il de la présence de ces Québécois dans la population? Sont-ils effectivement aussi nombreux? Ces chiffres correspondent-ils à leur nombre dans la population québécoise pour une représentation quantitative adéquate?

Le tableau 3 reprend les pourcentages de publicités représentant chacune des grandes familles ethniques et compare ces chiffres à ceux de la présence de ces familles dans la popula- tion québécoise. Ainsi, au Québec, il y avait, selon le recensement de 2001, 0,1\% de Québécois d'origine latinoaméricaine. Ces derniers ont été représentés dans $0,6 \%$ des publicités analysées. Ces pourcentages, étant plus ou moins semblables, nous affirmons que la représentation des Québécois d'origine latino-américaine dans les publicités analysées correspondait plus ou moins à la présence des ces Québécois dans la population. Par contre, le tableau 3 révèle qu'il y avait sur-représentation des Québécois d'origines asiatique et africaine. En effet, les Québécois d'origine africaine ont été représentés dans 15,2\% des publici- 
tés analysées alors qu'ils n'étaient que $2,1 \%$ dans la population toujours selon le recensement de 2001. Même constat pour les Québécois d'origine asiatique qui ont été représentés dans $5,9 \%$ des publicités analysées alors que leur nombre étaient de 1,7\% dans la population. Nous pourrions affirmer la même chose, mais dans une moindre mesure, pour les Québécois d'origines européenne ou moyenorientale qui ont également été sur-représentés dans les publicités analysées par rapport à leur présence dans la population québécoise. Cependant, les Québécois d'origine nord-américaine, autres que ceux dit "de souche» ont été sous-représentés dans les publicités analysées. Nous avons jugé bon ici de donner des chiffres plus spécifiques pour montrer que les Québécois d'origines amérindienne et ceux d'origine anglophone ont été représentés dans $0,4 \%$ des publicités analysées alors que leur présence dans la population du Québec étaient respectivement de $1,8 \%$ et $3,1 \%$ selon le recensement de 2001 .

\section{La représentation qualitative de la diversité culturelle québécoise dans les publicités télévisées francophones}

Les premiers résultats de notre recherche semblaient donc confirmer ce que les chercheurs américains et canadiens avaient observé, soit qu'une plus large place est maintenant faite aux personnes d'origines ethniques différentes dans les médias. Par ailleurs, à quoi ressemble cette place? Comment sont représentés qualitativement parlant les Québécois d'origines ethniques différentes dans la publicité télévisée francophone? Pour répondre à ces questions, nous avons analysé les éléments suivants lors d'un second visionnement des mêmes publi- cités: qu'est-ce qui était présenté dans la publicité et qui se rapportait à la diversité culturelle (une image, un son, un personnage)?; quel était le produit annoncé, qui était l'annonceur?; quel public était, selon nous, ciblé par l'annonceur?; combien de personnages composaient la publicité?; quelle était leur origine ethnique?; quel était leur sexe?; quelle était leur occupation et quel était leur rôle dans la publicité (secondaire, principal ou de figuration)?

Une fois toutes les publicités analysées selon ces éléments, nous avons comparé nos résultats à la grille de Colfax et Sternberg (1972) permettant l'identification de stéréotypes raciaux dans la publicité. Ces chercheurs ont en effet défini neuf types de publicités perpétuant des stéréotypes raciaux dans leurs représentations de la diversité culturelle:

The ad-black as celebrity est une publicité où le produit est associé à une personnalité d'origine ethnique différente connue et aimée du grand public. C'est, selon Colfax et Sternberg, un des moyens le moins controversé pour représenter la diversité culturelle;

The token ad-black présente un personnage d'origine ethnique différente à travers une masse de personnages natifs. Un peu de visibilité est ainsi donnée à la diversité culturelle, mais c'est rapidement le groupe des natifs qui l'emporte visuellement. Ceci a pour effet de renforcer le stéréotype que les natifs dominent les personnes d'origines ethniques différentes;

The all-races-together ad est une publicité présentant plusieurs personnages d'origines ethniques différentes à la fois ce qui évite de mettre l'accent sur une origine ethnique en particulier. Ici, aucun groupe ne peut être identifié comme dominant;

The non-commodity ad est une publicité représentant la diversité 
culturelle qu'à travers des consommateurs. La diversité culturelle n'est jamais alors représentée comme des personnes étant capables d'être également des producteurs de biens et services;

The exotic ad est une publicité où on fait appel aux stéréotypes «classiques» pour représenter la diversité culturelle dans ce qu'elle a de plus exotique. Par exemple, on y voit un Mexicain faisant la sieste ou un Afro-américain travaillant dans les champs de coton. Le prétexte est de représenter ces personnes d'origines ethniques différentes via leurs traditions, leur folklore ou leur histoire;

The black image as child-image est une publicité représentant la diversité culturelle via un enfant souvent accompagné d'un adulte à la peau blanche. Cela a pour effet de renforcer le stéréotype que les hommes blancs dominent sur les hommes d'origines ethniques différentes puisque ces derniers, étant représentés par un enfant, ne sont pas menaçants par leur jeune âge;

The black image as female est une publicité représentant la diversité culturelle via la féminité. Comme pour les publicités avec des enfants d'origines ethniques différentes, les femmes, étant considérées comme plus "accommodantes», sont plus souvent choisies pour représenter les différentes origines ethniques;

The ad-black as loser est une publicité représentant la diversité culturelle dans des situations de pauvreté ou d'échec. Selon Colfax et Sternberg, les publicités illustrant la pauvreté et la misère utilisent de manière disproportionnée des modèles d'origines ethniques différentes;

The black worker - in context est une publicité présentant un personnage d'origine ethnique différente occupant un emploi, mais sans haute stature.
Nous avons observé, comme Colfax et Sternberg, que les publicités représentant la diversité culturelle sont surtout des messages annonçant des produits de consommation. Plus spécifiquement, nous avons relevé plusieurs publicités faisant la promotion de produits corporels, automobiles et alimentaires. Par contre, nous avons observé un type de produit que Colfax et Sternberg n'avaient pas décelé dans leurs recherches soit celui des programmes gouvernementaux. Cependant, même dans les publicités annonçant ces services, les Québécois d'origines ethniques différentes tenaient presque toujours des rôles de consommateurs et non pas de producteurs en plus d'occuper majoritairement l'arrière plan (rôle secondaire ou de figuration). Par ailleurs, comme Colfax et Sternberg, nous avons observé que les Québécois d'origines ethniques différentes sont rarement présentés seuls à l'écran. En effet, cinq publicités de notre corpus mettaient en scène uniquement un personnage d'origine ethnique différente. Plus souvent qu'autrement, la diversité culturelle était donc noyée dans un océan de Québécois ou, dans quelques autres cas, elle était noyée dans une marrée de Québécois aux origines ethniques les plus diverses. Nos premières observations s'inscrivaient donc dans la même veine que les travaux de Colfax et Sternberg quant à la perpétuation de stéréotypes raciaux dans la publicité pour ce qu'ils ont appele The non-commodity ad, The token ad-black et The all-races-together ad.

Nous avons également observé de nouveaux genres de publicités qui, selon nous, entretiennent des stéréotypes raciaux. Quelques-unes de ces publicités avaient déjà été relevées par Colfax et Sternberg, mais elles semblent avoir gagné en popularité depuis leurs recherches puisqu'elles étaient nombreuses dans notre corpus. Ce 
sont tout d'abord les publicités que les deux chercheurs ont nommé The exotic $a d$ où la représentation folklorique des diverses origines ethniques passe pour nécessaire puisque l'on y fait la promotion d'un endroit exotique. Nous avons en effet analysé plusieurs publicités invitant les Québécois à choisir Cuba ou toute autre île des Caraïbes comme destination vacances où nous avons décelé des stéréotypes raciaux évidents. Également, nous avons dénombré plusieurs publicités que Colfax et Sternberg ont surnommé The ad-black as celebrity, soit des messages présentant des célébrités issues de l'immigration pour annoncer un produit. Cette pratique était bien présente dans les publicités que nous avons analysées. Nous avons aussi identifié une nouvelle forme publicitaire prisée par les annonceurs et le public québécois. Ce sont les publicités usant de l'humour qui semblent ridiculiser les stéréotypes raciaux, mais où ces derniers sont bien plus souvent, quant à nous, renforcés. Par exemple, un message de Vidéotron tentait de démontrer que les antennes satellites que propose la concurrence ne sont pas aussi efficaces que le câble; vaut alors mieux les utiliser comme wok dans la cuisine! Un personnage aux origines asiatiques montrait alors comment réussir un «chop suey» à l'aide d'une antenne satellite. Le personnage, à l'accent prononcé, criait et riait sans raison en faisant sauter les légumes. La caricature faisait rire, mais elle entretenait le stéréotype que les Asiatiques réagissent de façon disproportionnée, qu'ils sont bons pour faire la cuisine, qu'ils ne s'expriment pas clairement en français, etc. Nous jugeons que cette nouvelle forme publicitaire utilisant l'humour relève de ce que les plus récents auteurs appellent le nouveau racisme ou le racisme démocratique dont nous avons déjà fait mention dans cet article.
Figure 1: Extrait de la publicité de Vidéotron

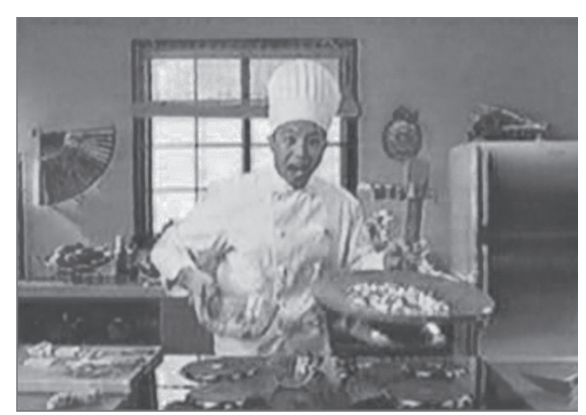

Source: publicité diffusée à la télévision québécoise en 2003.

Par ailleurs, nos résultats ne nous ont pas permis d'observer, de manière significative, les publicités que Colfax et Sternberg ont nommé The black worker - in context, The black image as female et The black image as child-image. En effet, les Québécois d'origines ethniques différentes présentés dans les publicités analysées occupaient toutes sortes de métier, du médecin à la femme de maison, et il n'y avait pas une représentation disproportionnée de femmes et d'enfants. À cet effet, nous avons comparé nos résultats quant au genre et à l'âge des personnages d'origines ethniques présentés dans les publicités à la pyramide des âges du Québec pour la même période et la représentation de femmes et d'enfants correspondait à la population québécoise.

\section{Les limites de la recherche}

La principale limite à cette recherche était l'identification des origines ethniques. Il est probable que des personnages représentant une origine ethnique ont été mal identifiés ou oubliés parce que leur traits physionomiques ressemblaient à ceux des Québécois. Par exemple, comment différencier un Nord-américain d'un Européen? Bien souvent des indices importants comme les accents, 
les prénoms et noms des personnages, le contexte, le décor et/ou l'environnement présenté, l'histoire racontée, les habillements,... nous permettaient de croire à la représentation de Québécois d'origines ethniques différentes. Il demeure que les origines ethniques les plus facilement identifiables à la télévision sont les minorités visibles. C'est peut-être pour cette raison que nous avons décelé plus de personnages d'origines africaine et asiatique lors de notre étude. De plus, il a été difficile de dénombrer exactement le nombre de personnages dans certaines publicités parce des messages présentaient des foules ou encore parce que les personnages se ressemblaient énormément (les publicités présentant des bébés en sont un bon exemple).

Il ne faut pas non plus passer sous silence l'incidence du choix du média qu'est la publicité par rapport à d'autres genres communicationnels. En effet, notre objet d'analyse, de par ses objectifs (vendre, susciter l'intérêt,...), ses prérogatives (faire passer un message en 30 secondes, ...), ses codes (faire appel aux raccourcis mentaux que sont les stéréotypes,...) a assurément teinté nos résultats.

Enfin, la provenance de la conception des publicités n’a pas été analysée. Ainsi, dans le corpus, des messages ont été produits par des agences québécoises tandis que d'autres ont probablement été produits par des agences internationales où le traitement et la notion de la diversité culturelle ne sont pas les mêmes. Par exemple, une publicité faisant la promotion de Cuba et produite par des Cubains ne peut pas avoir la même connotation que si elle est produite par des Québécois. Les agences n'ont pas été identifiées parce que cela aurait impliqué un travail dont l'ampleur dépassait le cadre de notre recherche.

\section{Le portrait de la publicité type représentant la diversité culturelle au Québec}

En conclusion de notre recherche, nous nous sommes permis de brosser le portrait de la publicité type représentant la diversité culturelle au Québec. Celle-ci présente un homme à la peau noire ayant un rôle secondaire et se trouvant au milieu d'une masse de Québécois dit «de souche». Cette publicité annonce de la nourriture, un produit pour le corps, une voiture ou un programme gouvernemental.

Notre étude, malgré ses limites, démontre qu'il y a lieu d'investiguer, au Québec, sur la question de la représentation de la diversité culturelle dans les médias francophones parce que des groupes sont sur-représentés et que d'autres sont sous-représentés et parce que des représentations entretiennent des stéréotypes raciaux. Enfin, nous avons observé une absence de représentation de la diversité culturelle par le son. Pourquoi, en effet, une publicité n'est pratiquement jamais narrée par un Québécois d'origine ethnique différente où on pourrait entendre les couleurs de son accent sans qu'il n'y ait un lien à faire avec le produit annoncé? Cette dernière observation qui n'a jamais été discutée par les chercheurs et auteurs mériterait, selon nous, d'être investiguée.

\section{Ouvrages cités}

COLFAX, J. David et Susan Frankel STERNBERG. "The Perpetuation of Racial Stereotypes: Blacks in Mass Circulation Magazine Advertisements", Public Opinion Quarterly, 1972, p. 8-18.

COTTLE, Simon. Ethnic minorities and the media: changing cultural boundaries, Buckingham, Open University Press, 2000. 
CULLEY, James-D. et Rex BENNETT. "Selling Women, Selling Blacks», Journal of Communication, automne, 1976, p. $160-174$.

FLERAS, Augie et Jean LOCK KUNZ. Media and minorities, Toronto, Thompson Educational, 2001.

FLERAS, Augie. Framing Diversity: Biased Coverage or Coverage that is Biasing?, communication présentée au colloque annuel de l'Association canadienne de communication à l'Université York de Toronto le 1er juin 2006.

MAHTANI, Minelle. La représentation des minorités: les médias canadiens et l'identité des groupes minoritaires, [En ligne], 2001, http://canada.metropolis.net/events/ethnocultural/publications/mahtani_f.pdf (Page consultée le 6 mars 2007).

SHUEY, Audrey M., Nancy KING et Barbara GRIFFITH. "Stereotyping of Negroes and Whites: An Analysis of Magazine Pictures», Public Opinion Quarterly, vol. 17, été, 1953, p. 281-287.

TREMBLAY, Pierre-André. La discrimination envers les minorités visibles au Québec: Le regard des sciences sociales. Chicoutimi, Chaire d'enseignement et de recherche interethniques et interculturels, Université du Québec à Chicoutimi, 1993. 